\title{
Negative Performance Evaluation in the Imposter Phenomenon: Content, Cause, and Authenticity
}

\author{
Stephen Gadsby ${ }^{1}$ \& Jakob Hohwy \\ Cognition \& Philosophy Laboratory, School of Philosophical, Historical \& International \\ Studies, 20 Chancellors Walk, Monash University, Clayton, VIC 3800, Australia.
}

\begin{abstract}
Intro: The imposter phenomenon (IP) is associated with a bias towards negative evaluation of one's own performance. In this study, we present a novel paradigm to explore the relationship between IP and performance evaluation. We employ this paradigm to test for different forms of bias associated with IP, including a bias towards selecting negative feedback about one's own performance. We also explore the relationship between IP and performance evaluation in light of the related constructs of depression and low self-esteem.
\end{abstract}

Method: Our sample ( $\mathrm{n}=163)$ included graduate students living in the United Kingdom, United States, and Europe, recruited from the online platform Prolific. Participants solved several reasoning problems and reported their confidence in their performance, during the test. After receiving some (self-selected) feedback on their performance, participants estimated their performance, both objectively (how many problems they correctly solved) and comparatively (percent of participants they outperformed).

Results: Participants high in IP evaluated their performance more negatively, in all three forms. This bias in performance evaluation was not associated with a bias towards selecting negative feedback. When controlling for the effect of depression and low self-esteem, IP only significantly correlated with comparative performance evaluation.

Conclusion: Participants high in IP negatively evaluate their performances in anonymous online settings. This validates the use of such paradigms for exploring the relationship between IP and performance evaluation bias. However, greater attention should be paid to the different forms of performance evaluation, as well as the association with similar constructs, such as depression and low self-esteem.

Keywords: Imposter Phenomenon; Imposter Syndrome; Motivated Reasoning; SelfDeception; Biased Reasoning

\section{Acknowledgments:}

Thanks to the members of the Cognition and Philosophy and Dijkerman labs for helpful discussion; Manja Engel, Ryan McKay, and Jennifer Windt for written feedback; Simon van Baal and Jarl Kampen for analysis and coding advice; and Andrew Gadsby for figure design. This study was funded by an Australian Government Research Training Program (RTP) Scholarship (S.G), a Monash University Postgraduate Publications Award (S.G.), a Fonds Wetenschappelijk Onderzoek (FWO) Postdoctoral Fellowship (1267022N) (S.G), and the Arts Faculty at Monash University (J.H).

\footnotetext{
${ }^{1}$ Current Address: Department of Philosophy, University of Antwerp, Antwerp, Belgium.
} 


\section{Introduction}

In the imposter phenomenon (IP), successful and intelligent people believe that they are incompetent and fear being exposed as such (Clance \& Imes, 1978). A key feature of IP is negative evaluation of one's own performance. People high in IP expect themselves to perform poorly in upcoming exams, and judge themselves as having performed poorly, after completing exams (Cozzarelli \& Major, 1990). This bias is considered to be a crucial feature of, and driving force behind, IP (Clance, 1985). By judging themselves to have performed poorly, individuals high in IP reinforce their belief in their own incompetence; by expecting themselves to perform poorly, they exacerbate their fears of being exposed as imposters.

A recent theory suggests that IP involves motivated reasoning (Gadsby, 2021). The theory draws from evidence on defensive pessimism, which illustrates how negative thinking is often employed for the goal of self-motivation (Norem \& Cantor, 1986). It claims that people high in IP are motivated to believe that they lack ability (for example, that they are unintelligent), because this belief motivates them to work harder ("Because I am not intelligent, I will have to work harder in order to succeed"). According to this theory, people high in IP negatively evaluate their own performance to maintain these negative but motivationally beneficial beliefs.

The literature on motivated reasoning suggests several strategies that underpin motivated reasoning (Bénabou \& Tirole, 2002). One such strategy involves selectively exposing oneself to evidence in a biased manner (Frimer et al., 2017). For example, in a recent study participants were motivated to positively judge their performance in a general knowledge test (Solda et al., 2019). After completing the test, they were allowed to select questions for which they would receive feedback (whether they answered correctly or not). Participants motivated to judge themselves positively selected more questions that they had answered correctly. In other words, they chose to receive more positive feedback, biasing themselves towards (over)confidence in their own performance. The motivated reasoning account of IP predicts that people high in IP will exhibit the opposite pattern of bias: because they are motivated to negatively judge themselves, they will disproportionately seek out negative evidence regarding their performance. This prediction is consistent with the clinical observation that people high in IP pay inordinate attention to evidence regarding performance failure, while ignoring evidence of success (Clance \& OToole, 1987).

While the motivated reasoning account of IP offers some useful predictions about the cause of negative performance evaluations associated with IP, there are competing accounts. For example, some suggest that people who score high in measures of IP do not genuinely believe that they are incompetent and judge themselves as performing poorly. Instead, they claim that negative performance evaluations associated with IP are a social strategy (Leary et al., 2000; McElwee \& Yurak, 2007), conducted for interpersonal benefits, such as lowering others' expectations, conveying a sense of modesty, protecting one's image in the face of failure, and eliciting support from others (Leary et al., 2000, p. 752). While Leary et al. leave open the possibility that "true imposters"-who genuinely believe that they are inadequateexist, they nevertheless claim that "people who score high on measures of [IP] are strongly motivated by self-presentational concerns" (p. 753; see also: Leonhardt et al., 2017). On this account, IP does not qualify as a form of motivated reasoning, as the relevant beliefs and reasoning biases are not authentic. Rather, people high in IP (consciously) feign such attitudes.

Another important issue regarding the relationship between IP and negative performance evaluation relates to differences in the form of evaluations. Previous research has focused on 
the difference between prospective evaluation (how well one judges that they will perform, in an upcoming task) and retrospective evaluation (how well one judges that they performed, after completing a task) (Cozzarelli \& Major, 1990). However, an underexplored form of selfevaluation involves how one judges their performance, at the time of performing. Another important distinction is between objective performance evaluations (how well one judges that they performed) and comparative performance evaluation (how well one judges that they performed, compared to their peers). To better understand negative performance evaluations in IP, we must understand their different forms.

One final important aspect of the relationship between IP and performance evaluation pertains to the relationship between IP and associated traits, namely, low self-esteem and depression. IP highly correlates with low self-esteem and depression (Cozzarelli \& Major, 1990; McGregor et al., 2008), both of which are associated with a bias towards negative selfevaluation (Campbell \& Fairey, 1985; Kovacs \& Beck, 1978). Consequently, it is worth exploring whether and which forms of performance evaluation bias are associated with these related traits.

This study investigated the relationship between IP and performance evaluation bias. To do so, we adapted an experimental paradigm from the motivated reasoning literature, wherein participants were required to solve a set of reasoning problems and evaluate their own performance (Schwardmann \& Van der Weele, 2019; Solda et al., 2019). If, as suggested, the negative performance evaluations associated with IP are feigned, then anonymous online tasks such as these (which remove the possibility for social gain by feigning negativity) may not be appropriate for measuring the relevant biases.

The study had four aims. The first aim was to test whether anonymous online intelligence tests, of a form used to research motivated reasoning, could be employed to examine the performance evaluation bias associated with IP.

The second aim was to explore the relationship between IP and different forms of performance evaluation bias. After solving each problem, participants were required to rate their confidence in the accuracy of their solution, indicating an ongoing form of performance evaluation. We also measured two forms of retrospective performance evaluation: objective and comparative. After receiving some self-selected feedback on their performance (see below), participants were required to estimate their total accuracy on the set of problems (objective performance evaluation), as well as the percent of other participants that they outperformed (comparative performance evaluation).

The third aim was to test whether participants high in IP would exhibit a bias towards selecting more negative feedback regarding their own performance. To test this hypothesis, we allowed participants to select between one and four of their solutions to receive feedback on, measuring whether those higher in IP would show a bias towards receiving feedback for problems that they were less confident of (i.e. solutions that would more likely be false, constituting negative feedback).

The fourth aim was to explore the relationship between IP, depression, low self-esteem and negative performance evaluations. To do so, we measured participants' levels of depression and self-esteem and measured the correlation between these factors and the measured forms of performance evaluation bias.

We constructed two hypotheses. First, contrary to the suggestion that the negative performance evaluation associated with IP is feigned for social benefit, we expected that the relationship between IP and negative performance evaluation would still hold in this anonymous online setting. We further expected that negative bias would be found in all three forms of performance evaluation (ongoing, objective, and comparative). Second, we expected 
that, in line with the motivated reasoning account, IP would be associated with a search bias, such that those high in IP would select more negative feedback regarding their performance.

\section{Materials and Methods}

\subsection{Ethics}

This study was approved by Monash University Human Research Ethics Committee (MUHREC Project ID: 25939). Participants were informed about the design and purpose of the study (see supplementary material) and provided informed consent before taking part.

\subsection{Participants}

201 participants were recruited online, through Prolific (https://www.prolific.co/). We recruited participants between the ages of 18 and 65, who were currently residing in United Kingdom, United States, and continental Europe, and were currently enrolled in graduate studies (e.g. MA/MSc/MPhil/PhD). Graduate students were selected as this demographic exhibit high levels of IP and often compare themselves with one another to gauge ability. All participants were fluent in English and reported no current or prior diagnosis of a neurological or psychiatric condition.

Participants were compensated a base rate of $£ 1.70$. They were also given a bonus, designed to incentivise effort. For each participant, 2 of their answers were randomly selected and they were paid £o.80 for each correct answer. Including bonuses, the total payment, per participant, fell between $£ 1.70$ and $£ 3.30$.

Participants (5) were excluded when they failed one of two measures of effort, either scoring less than 3 out of 16 questions correctly or failing a question designed to test effort ("What is the sixth month of the year"). Participants (31) were also excluded for failing one of two measures of general interest in their score on the reasoning task, either for choosing not to see their final score and the set of answers (13) or selecting 4 answers in a row (e.g. 1, 2, 3,4$)$ when selecting feedback (18). We reasoned that those who showed no interest in the outcome of the task would thoughtlessly select questions to receive feedback for, undermining the data on search bias. Given the risks of low-quality data associated with online studies, these exclusion principles were chosen to filter out as much low-quality data as possible (at the risk of losing some high-quality data); the pre-exclusion data set is openly available (see below). The final sample included 163 participants (93 females; 70 males; M age $=25.4 ; \mathrm{SD}: 4.4)$.

\subsection{Material and Procedure}

The dataset and code for this project is available on the Open Science Framework page (https://osf.io/3ng6e/). Data was collected through Qualtrics (http://www.qualtrics.com/). After providing informed consent, participants filled out a demographic questionnaire, completed the main task, and completed three questionnaires (CIPS, RSS, BDI-II, provided in that order, see below for details). They were then given the opportunity to return directly to Prolific or view their total score and the complete set of answers.

\subsubsection{Main Task}

In the main task, participants were required to complete 16 reasoning problems ("designed to test your intelligence"). Problems were taken from the international cognitive reasoning ability resource (Condon \& Revelle, 2014) and the test of figural analogies (Blum et al., 2016). These included verbal reasoning, letter and number series, three-dimensional 
rotation, matrix reasoning, and figural analogy problems (for problem set, see supplementary material).

Participants were allowed two minutes to solve each problem. After providing a solution, they were required to report their confidence in it ("How confident are you that you answered correctly?") on a scale from o ("not confident at all") to 100 ("completely confident”).

After solving the entire problem set, participants were shown each of the problems, along with the confidence that they reported in their solutions. They were asked to select between one and four of the problems to receive feedback for: "You may now check whether some of your answers were correct. You will only be informed about whether each answer was correct, you will not be given the answers to the questions".

After receiving feedback for the problems that they selected, they were asked to evaluate their performance. First, they estimated how many problems $(0-16)$ that they had solved correctly. Second, they estimated the percent of other participants (presented as graduate students in the UK, US, and Europe) that they outperformed (0\%-100\%).

\subsubsection{Questionnaires}

After completing the main task, participants answered three questionnaires. In order to measure IP, we employed the Clance Imposter Phenomenon Scale (CIPS) (Clance, 1985), which is the most commonly employed and exhibits the strongest validity and reliability $(\mathrm{a}=.91$; Holmes et al., 1993$)$.

To measure self-esteem, we employed the Rosenberg Self-esteem Scale (Rosenberg, 1965) (RSS), a widely used measure of global self-esteem ( $\mathrm{a}=.94$; Kolligian \& Sternberg, 1991) which has been found to correlate with the IP, as measured by the CIPS (Sonnak \& Towell, 2001).

To measure depression, we employed the Beck Depression Inventory II ( $\mathrm{a}=.93$, Beck, Steer, \& Brown, 1996) (BDI-II), a commonly used scale to measure depression, which has also been found to correlate with IP, as measured by the CIPS (McGregor et al., 2008).

\subsection{Data Preparation and Analysis}

\subsubsection{Data Preparation}

CIPS, BDI-II, and RSS scores were calculated by summing the responses to each statement of the respective questionnaires. Participants' total accuracy was recorded on the reasoning problems, as well as their ongoing reported confidence for each of their solutions, and their retrospective performance evaluations. Based on these scores, four dependent variables were calculated:

Ongoing evaluation bias was calculated by subtracting a participant's score (\%) from the mean of their (ongoing) confidence ratings. Negative values represent a negative bias (being less confident in one's performance than is warranted), while positive values represent a positive bias (being more confident than is warranted);

Objective evaluation bias was calculated by subtracting participants' score (\%) from their estimated score (number of questions answered correctly, converted into \%). Negative values indicate a negative bias (underestimating one's own score), while positive values indicate a positive bias (overestimating it); 
Comparative evaluation bias was calculated by subtracting the percent of the sample a participant outperformed, from the percent that they estimated themselves to have outperformed (see the OSF project for the syntax used to calculate rank). Negative values indicate a negative bias (underestimating one's own comparative performance), while positive values indicate a positive bias (overestimating it);

Search bias was calculated by subtracting the mean confidence (\%) of the participants' searched answers from the overall mean confidence (\%). A negative value represents a negative bias (participants choosing feedback for answers that they were less confident of, i.e. they believed were more likely to be inaccurate), while a positive value represents a positive bias (participants choosing feedback for answers that they were more confident of, i.e. they believed were more likely to be accurate).

\subsubsection{Analysis}

All analysis was conducted using IBM SPSS Statistics (27.0.0.0) and JASP (JASP Team, 2020). To test our two hypotheses, we conducted between-group analyses, comparing participants who scored high in IP against those who scored low along all three measures of performance evaluation bias (hypothesis 1) and search bias (hypothesis 2). Following the approach of previous studies (Ferrari \& Thompson, 2006), we split the data set into two groups, based on their CIPS scores: the top-third of participants (high imposter group; $n=54$; range $=70-91 ; \mathrm{M}=79.37, \mathrm{SD}=6.54$ ) and the bottom-third (low imposter group; $\mathrm{n}=54$; range $=26-58 ; \mathrm{M}=49.91, \mathrm{SD}=6.67)$. These ranges are consistent with the suggested CIPS cutoff between "impostors" and "non-impostors" (61) (Holmes et al., 1993).

To test for a between-group difference, we conducted independent sample t-tests, using each of the dependent variables: ongoing evaluation bias, objective evaluation bias, comparative evaluation bias, and search bias, as well as participant accuracy. Normality was assessed with the Shapiro-Wilk test. In both the low-IP and high-IP groups, objective evaluation bias was significantly non-normal, low-IP: $\mathrm{D}(54)=.953, \mathrm{p}=.033$; high-IP: $\mathrm{D}(54)=.947, \mathrm{p} .=.019$. In the low-IP group, ongoing evaluation bias was also significantly non-normal, D(54) $=.956$, p. $=.047$. However, in each case the extent of kurtosis and skewness fell within an acceptable range $( \pm 2)$ (George \& Mallery, 2010). Homogeneity of variance was assessed with Levene's test. One-directional hypotheses were specified (the high-IP group exhibiting more negative evaluations than the low-IP group), so one-tailed p-values were reported. Null-findings were followed up with Bayesian analysis (Dienes, 2014). We calculated Bayes factors $\left(B F_{O 1}\right)$ using the default priors set by JASP (Cauchy prior, $\mathrm{r}=0.707$ ).

To explore the relationship between IP, depression, self-esteem, and negative performance evaluation in our (complete) sample, we performed Pearson correlations using the CIPS, BDI-II, RSS scores and the four measures of bias. Additionally, we report semi-partial correlations to measure the relationship between IP and evaluation bias, while statistically controlling for the effect of BDI-II and RSS. For each of these variables the assumption of normality was tested. BDI-II, D(163) $=.931$, $\mathrm{p}<.001$, Objective evaluation bias, $\mathrm{D}(163)=.971$, $\mathrm{p}=.002$, and Search bias $\mathrm{D}(163)=.976, \mathrm{p}=.016$ were all found to be significantly non-normal (according to the Shapiro-Wilk test). However, both skewness and kurtosis fell within an acceptable range $( \pm 2)$ for all three variables (George \& Mallery, 2010). Inspection of scatterplots indicated no violation of linearity and homoscedasticity assumptions. Onedirectional directions were expected (CIPS predicting negative bias), so one-tailed p-values were reported.

\section{Results}




\subsection{Between-Group Analysis}

Four independent sample t-tests revealed the following: there was a significant difference in ongoing evaluation bias between the high-IP $(\mathrm{M}=-2.42, \mathrm{SD}=17.45)$ and low-IP $(\mathrm{M}=7.13$, $\mathrm{SD}=16.49$ ) groups; $\mathrm{t}(106)=2.92, \mathrm{p}=.004, \mathrm{~d}=0.563$ (figure $1 \mathrm{a}$ ). There was a significant difference in objective evaluation bias between the high-IP $(\mathrm{M}=-8.22, \mathrm{SD}=13.12)$ and low-IP $(\mathrm{M}=0.35, \mathrm{SD}=14.02)$ groups; $\mathrm{t}(106)=3.28, \mathrm{p}=.001, \mathrm{~d}=0.631$ (figure $1 \mathrm{~b})$. There was a significant difference in comparative evaluation bias between the high-IP $(\mathrm{M}=-13.77$, $\mathrm{SD}=32.61)$ and low-IP $(\mathrm{M}=2.93, \mathrm{SD}=33.34)$ groups; $\mathrm{t}(106)=2.63, \mathrm{p}=.010, \mathrm{~d}=0.506$ (figure 1c). This means that participants in the high-IP group were significantly more negative in their ongoing, comparative, and objective evaluations, than those in the low-IP group.

There was no significant difference in search bias between the high-IP (M=-12.64, $\mathrm{SD}=24.40)$ and low-IP $(\mathrm{M}=-13, \mathrm{SD}=23.6)$ groups; $\mathrm{t}(106)=-.08, \mathrm{p}=.938, \mathrm{~d}=-0.015, B F_{O 1}$ $=4.894$ (figure 1d). There was also no significant difference between high-IP $(\mathrm{M}=-61.11$, $\mathrm{SD}=18.60)$ and low-IP $(\mathrm{M}=-63.43, \mathrm{SD}=14.69)$ groups in terms of actual performance accuracy; $\mathrm{t}(106)=-0.718, \mathrm{p}=.475, \mathrm{~d}=-0.138, B F_{O 1}=3.897$. The results of the Bayesian analysis indicate moderate evidence for these two null hypotheses (Jeffreys, 1998)
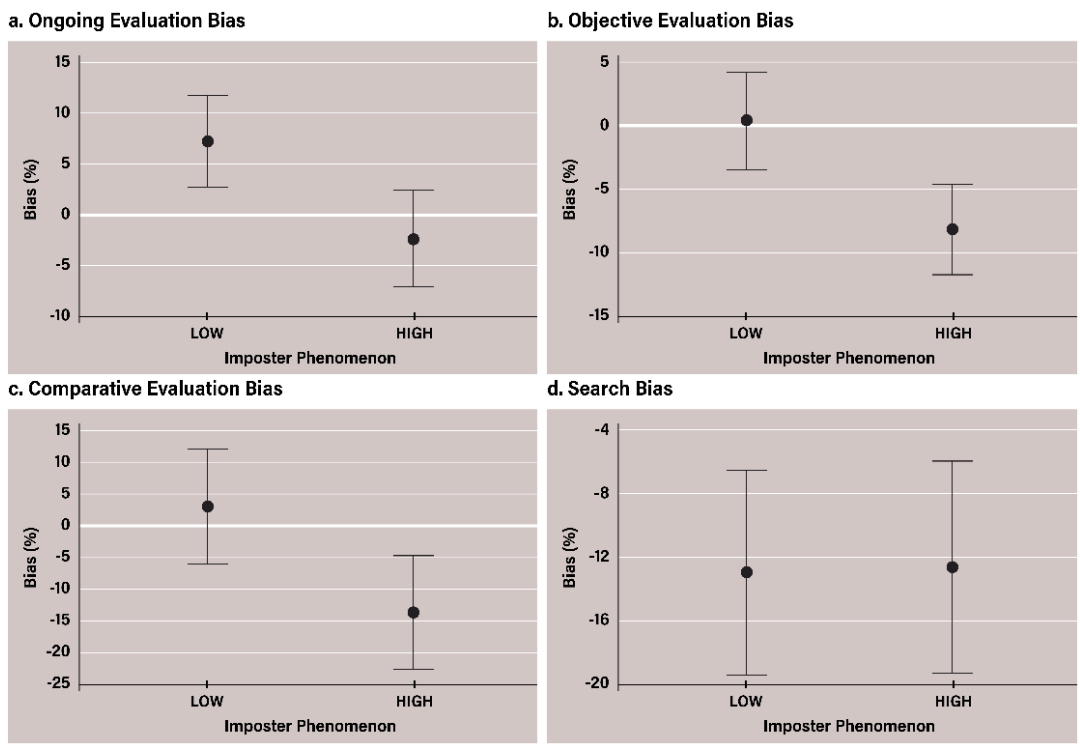

Figures 1a, 1b, 1c, 1d: Between-group comparison of ongoing evaluation bias, objective evaluation bias, comparative evaluation bias, and search bias. Error bars represent 95\% CI

\subsection{IP, Self-Esteem, Depression, and Performance Evaluation Bias}

Consistent with the between-group finding CIPS negatively correlated with Ongoing evaluation bias ( $r=-.22, p=.002)$, Objective evaluation bias $(r=-.22, \mathrm{p}=.003)$ and Comparative evaluation bias $(r=-.21, p=.004)$, while the correlation with Search bias was not significant ( $\left.r=-.03, p=.377, B F_{O 1}=9.718\right)$.

CIPS also positively correlated with BDI-II $(r=.57, p<.001)$ and negatively correlated with RSS ( $r=-.67, p .<.001)$. This means that those who scored higher on IP also scored higher in depression and lower on self-esteem.

When controlling for the relationship between BDI-II+RSS, CIPS was found to significantly correlate with Comparative evaluation bias $(r=-.17, p=.029)$. However, the analysis uncovered no significant relationship between CIPS and Ongoing evaluation bias $(r=-.09$, $p=.264)$ or Objective evaluation bias $(r=.10, \mathrm{p}=.197)$. 


\section{Discussion}

To understand the relationship between IP and negative performance evaluation, experimental paradigms are needed to measure different forms of performance evaluation. This study presents a novel experimental paradigm to accomplish this. In an online setting, participants were required to solve a set of reasoning problems while evaluating each of their solutions, and, after receiving some self-selected feedback, estimate their own performance.

We constructed two hypotheses. First, that in an anonymous online setting-where there was no social benefit to feigning negativity-participants high in IP would exhibit a bias towards negative performance evaluation, in each of the three forms (ongoing evaluation, objective evaluation, and comparative evaluation). Second, that participants high in IP would also exhibit a negative search bias, selecting feedback for solutions that they felt less confident in (i.e., problems they were more likely to have solved incorrectly).

The findings supported our first hypothesis. IP was associated with a bias towards negative estimates in each of the three forms of performance evaluation (ongoing, objective, and comparative). Those in the high IP group were more negative in their performance evaluation than the low IP group, despite performing equally well. Our findings did not support the second hypothesis, that IP would be associated with a negative search bias. There was no significant between-group difference in search bias between high and low IP groups, nor did IP did correlate with search bias. This null hypothesis was supported by the results of Bayesian analysis.

Regarding the exploratory aspect of the study-concerning the relationship between depression, low self-esteem, and performance evaluation bias-our findings were illuminating. IP correlated with the three forms of bias (ongoing evaluation, objective evaluation, and comparative evaluation), consistent with the between-group findings. IP also correlated with both depression and low self-esteem, consistent with previous findings (Cozzarelli \& Major, 1990; McGregor et al., 2008). Interestingly, when controlling for the effect of depression and self-esteem on performance evaluation bias, IP was only found to significantly correlate with comparative performance bias.

Our findings are consistent with those high in IP exhibiting bias in evaluation of their own performance. In our study, participants higher in IP were less confident in the accuracy of their solutions, while performing (ongoing performance bias), and more negative in their (retrospective) evaluation of their performance. Performance evaluations in this study were made anonymously, provided to a researcher who the participants had never met and would never meet; there was thus no social benefit for participants in pretending to negatively evaluate themselves. Contrary to suggestions that many, if not most, who score highly in measures of IP feign negative self-evaluation for social advantage, our results suggest that such biases are present in contexts where there is no social advantage to doing so.

However, we did not employ social setting as a manipulation in this study, and so our results are not quantitatively informative of the role of social setting in driving these factors. It might be that while some people who score high in IP feign negative evaluations, others do so genuinely, and the presence of this latter group was sufficient to drive the observed results (Leonhardt et al., 2017). Our findings do, however, suggest that anonymous online studies are a useful tool for exploring the association between IP and biased performance evaluation. These results also do not speak to the more general claim that performance evaluation biases associated with the imposter phenomenon are driven by some form of social considerations. Several strands of research suggest that human reasoning is highly sensitive to social consideration, in ways that are evident in anonymous online settings (Williams, 2020). This study only speaks to the restricted claim that these biases are not only socially driven but feigned. 
The effects of our exploratory analysis contribute to the body of evidence suggesting a link between depression, low self-esteem, and IP. They also suggest that researchers interested in the link between IP and performance evaluation bias should consider the effect that high levels of depression and low levels of self-esteem have on contributing to such bias. The finding that IP correlates with comparative performance evaluation bias, when controlling for self-esteem and depression, is also important. Previous research on performance evaluation bias in IP has focused on objective evaluations of performance (e.g. obtaining a certain grade) (Cozarelli \& Major 1990). However, classic accounts of IP emphasize its socially comparative nature. Those high in IP specifically downplay their abilities related to their peers, insisting that they are less capable and intelligent than them (thus meriting the "imposter" label) (Clance, 1985). Our findings are consistent with this characterisation and suggest that while comparisons may not be feigned for social benefit, they are of a socially comparative nature. Consequently, comparative evaluations may be a more appropriate target for understanding the specific forms of biased performance evaluation associated with IP.

Our results did not support the hypothesis that negative performance evaluation in IP is maintained by a bias in searching for evidence. While participants high in IP were more negative in their performance evaluations, they did not select more negative feedback than other participants. Negative performance evaluation may, instead, be underpinned by distinct strategies. For example, instead of seeking out negative feedback, those high in IP may exhibit biased memory recall, disproportionately remembering problems that they could not solve (Bénabou \& Tirole, 2002, p. 149). They might also be more biased in their interpretation of the feedback that they received, allowing negative feedback (incorrect solutions) to more strongly influence their judgments about performance (Lord et al., 1979). Future research should explore alternative strategies that might underpin the negative performance evaluation associated with IP. To do so, researchers might adapt other paradigms from the research into motivated reasoning (Van der Leer \& McKay, 2017). Bias in the selection of feedback may also still be associated with IP, though the present study was unable to discover it. For example, participants' curiosity regarding the accuracy of their solutions may have simply outweighed any alternative motivations to engage in biased information search.

\section{Conclusion}

In summary, our results showed that individuals high in IP exhibit a bias towards negative performance evaluation, in an anonymous online setting. This bias extends to different forms of performance evaluation, both during the task and retrospectively. However, the bias is not (necessarily) induced by a bias in the selection of feedback on performance. Further, when controlling for depression and low self-esteem, the relationship between IP and negative performance evaluation was only associated with one form of evaluation, comparison to others, suggesting that this form is the most appropriate target for future research. 


\section{References}

Bénabou, R., \& Tirole, J. (2002). Self-confidence and personal motivation. The quarterly journal of economics, 117(3), 871-915.

Blum, D., Holling, H., Galibert, M. S., \& Forthmann, B. (2016). Task difficulty prediction of figural analogies. Intelligence, 56, 72-81.

Clance, P. R. (1985). The impostor phenomenon: Overcoming the fear that haunts your success. Peachtree Pub Ltd.

Clance, P. R., \& Imes, S. A. (1978). The imposter phenomenon in high achieving women: Dynamics and therapeutic intervention. Psychotherapy: Theory, Research \& Practice, 15(3), 241.

Clance, P. R., \& OToole, M. A. (1987). The imposter phenomenon: An internal barrier to empowerment and achievement. Women \& Therapy, 6(3), 51-64.

Condon, D. M., \& Revelle, W. (2014). The International Cognitive Ability Resource: Development and initial validation of a public-domain measure. Intelligence, 43, 52 64.

Cozzarelli, C., \& Major, B. (1990). Exploring the validity of the impostor phenomenon. Journal of social and clinical psychology, 9(4), 401-417.

Dienes, Z. (2014). Using Bayes to get the most out of non-significant results. Frontiers in psychology, 5, 781.

Ferrari, J. R., \& Thompson, T. (2006). Impostor fears: Links with self-presentational concerns and self-handicapping behaviours. Personality and Individual differences, 40(2), 341-352.

Frimer, J. A., Skitka, L. J., \& Motyl, M. (2017). Liberals and conservatives are similarly motivated to avoid exposure to one another's opinions. Journal of Experimental Social Psychology, 72, 1-12. https://doi.org/10.1016/j.jesp.2017.04.003

Holmes, S. W., Kertay, L., Adamson, L. B., Holland, C., \& Clance, P. R. (1993). Measuring the impostor phenomenon: A comparison of Clance's IP Scale and Harvey's IP Scale. Journal of personality assessment, 60(1), 48-59.

JASP Team. (2020). JASP. In (Version 0.14.1)

Jeffreys, H. (1998). The theory of probability. OUP Oxford.

Kolligian, J., \& Sternberg, R. J. (1991). Perceived Fraudulence in Young Adults: Is There an'Imposter Syndrome'? Journal of personality assessment, 56(2), 308-326.

Leary, M. R., Patton, K. M., Orlando, A. E., \& Wagoner Funk, W. (2000). The impostor phenomenon: Self-perceptions, reflected appraisals, and interpersonal strategies. Journal of personality, 68(4), 725-756.

Leonhardt, M., Bechtoldt, M. N., \& Rohrmann, S. (2017). All impostors aren't alikedifferentiating the impostor phenomenon. Frontiers in psychology, 8, 1505.

Lord, C. G., Ross, L., \& Lepper, M. R. (1979). Biased assimilation and attitude polarization: The effects of prior theories on subsequently considered evidence. Journal of personality and social psychology, 37(11), 2098.

McElwee, R. O. B., \& Yurak, T. J. (2007). Feeling versus acting like an impostor: Real feelings of fraudulence or self-presentation? Individual Differences Research, 5(3), 201 220.

McGregor, L. N., Gee, D. E., \& Posey, K. E. (2008). I feel like a fraud and it depresses me: The relation between the imposter phenomenon and depression. Social Behavior and Personality: an international journal, 36(1), 43-48. 
Norem, J. K., \& Cantor, N. (1986). Defensive pessimism: Harnessing anxiety as motivation. Journal of personality and social psychology, 51(6), 1208.

Rosenberg, M. (1965). Society and the adolescent self-image. Princeton University Press.

Schwardmann, P., \& Van der Weele, J. (2019). Deception and self-deception. Nature Human Behaviour, 3(10), 1055-1061.

Solda, A., Ke, C., Page, L., \& Von Hippel, W. (2019). Strategically delusional. Experimental Economics, 1-28.

Sonnak, C., \& Towell, T. (2001). The impostor phenomenon in British university students: Relationships between self-esteem, mental health, parental rearing style and socioeconomic status. Personality and Individual differences, 31(6), 863-874.

Van der Leer, L., \& McKay, R. (2017). The optimist within? Selective sampling and selfdeception. Consciousness and cognition, 50, 23-29.

Williams, D. (2020). Socially adaptive belief. Mind \& Language. 